\title{
Las comunidades de helmintos del lenguado (Symphurus plagiusa) en la costa de Campeche, México
}

\section{The helminth communities of tonguefish (Symphurus plagiusa) of the Campeche coast, Mexico}

\author{
Abril Rodríguez-González* y Víctor M. Vidal-Martínez \\ Laboratorio de Parasitología, Centro de Investigación y de Estudios Avanzados, Instituto Politécnico Nacional- Unidad Mérida, Carretera Antigua a \\ Progreso Km. 6, 97310 Mérida, Yucatán, México \\ *Correspondencia: arodriguez@mda.cinvestav.mx
}

\begin{abstract}
Resumen. Se caracterizaron las comunidades componentes e infracomunidades de helmintos de Symphurus plagiusa en 37 puntos de muestreo de mayo a septiembre de 2003 en la costa de Campeche. Además, se exploraron posibles asociaciones estadísticas entre la abundancia de cada especie y los parámetros ambientales (salinidad, oxígeno, volumen de descarga de agua de ríos) mediante análisis de redundancia (RDA). Se examinaron 531 hospederos, cuyas comunidades componentes e infracomunidades fueron pobres en número de especies (máximos: 8 y $3 \pm 1$ respectivamente), pero no en individuos (16775 y $47 \pm 32$ ). La diversidad fue baja (componente: Simpson $=1$; infracomunidad: Brillouin $=0.24 \pm$ 0.11). La similitud en cada mes fue baja para componente (cualitativa, Jaccard: $0.25 \pm 0.34$; cuantitativa, $\%$ de similitud: $51 \pm 49$ ) e infracomunidad (cualitativa: $0.59 \pm 0.24$; cuantitativa: $46 \pm 24$ ). La similitud entre meses fue alta en componente (cualitativa: $0.63 \pm 0.28$; cuantitativa: $82 \pm 15$ ) e infracomunidad (cualitativa: $0.89 \pm 0.10$; cuantitativa: $77 \pm 21$ ). Hubo asociaciones significativas entre la abundancia de especies y el volumen de descarga de agua de los ríos entre meses, lo que sugiere que este parámetro influye fuertemente sobre la composición y estructura de estas comunidades.
\end{abstract}

Palabras clave: comunidades de helmintos, variación temporal, lenguado, golfo de México.

\begin{abstract}
The component communities and infracommunities of Symphurus plagiusa were characterized for 37 sampling points from May to September 2003 along the Campeche coast. Possible statistical associations were also studied between the abundance of each helminth species and environmental parameters (e.g. salinity, oxygen concentration, river discharge water volumes) using redundancy analysis (RDA). We examined 531 hosts, whose component communities and infracommunities were poor in species number (maximum: 8 and $3 \pm 1$ respectively); but not in number of individuals (16775 and $47 \pm 32$ ). Diversity was low (component: Simpson $=1$; infracommunity: Brillouin $=0.24 \pm 0.11$ ). Similarity within months was also low for both component communities (qualitative, Jaccard: $0.25 \pm 0.34$; quantitative, \% of similarity: $51 \pm 49$ ) and infracommunities (qualitative: $0.59 \pm 0.24$; quantitative: $46 \pm 24$ ). Similarity between months was high for component communities (qualitative: $0.63 \pm 0.28$; quantitative: $82 \pm 15$ ) and infracommunities (qualitative: 0.89 \pm 0.10 ; quantitative: $77 \pm 21$ ). There were significant associations between the abundance of species and the river water discharges between months, which suggest high influence of this parameter on the structure and composition of these helminth communities.
\end{abstract}

Key words: helminth communities, temporal variation, tonguefish, Gulf of Mexico.

\section{Introducción}

Una pregunta clave en ecología de comunidades de helmintos es si estas entidades son predecibles en el tiempo y espacio o si son sólo ensambles de especies al azar resultado de procesos de colonización independiente (Holmes, 1987; Vidal-Martínez y Poulin, 2003).

En el caso específico de las comunidades de helmintos parásitos de peces marinos, se ha argumentado que éstas son

Recibido: 12 enero 2006; aceptado: 12 septiembre 2007 más ricas en número de especies e individuos y con mayor predictibilidad que las de peces de agua dulce (Kennedy et al., 1986; Holmes, 1990; Marcogliese, 2001). Entre las explicaciones más persistentes a este patrón que se dan en la literatura está la mayor talla de los hospederos marinos y sus hábitos alimenticios generalistas (Kennedy et al., 1986), así como la diversidad diferencial de hospederos intermediarios en los ecosistemas marinos (Rhode, 1992; Vidal-Martínez y Poulin, 2003; Marques et al., 2006). Esto último implica que mientras los peces pelágicos tienden a presentar comunidades más pobres en número 
de especies, las comunidades de los bentónicos, como son los lenguados, tienden a una mayor riqueza (Khan, 2004).

Sin embargo, el análisis comparativo de la riqueza de especies de las comunidades de helmintos de lenguados demuestra que esta generalización es difícil de sostener. Por ejemplo, para la comunidad componente, Lile (1998) registró entre 6 y 10 especies de helmintos en Hippoglosoides platessoides en Noruega; Siddall et al. (1994) registraron 7 para la misma especie en el Reino Unido, y recientemente Schmidt et al. (2003) en Alemania, 30 en Platichthys flesus. Sin embargo, Marques et al. (2006) registraron una gran variabilidad en la riqueza de especies en lenguados de Portugal; desde una en Synaptura lusitanica hasta 15 en Microchirus azevia. En la parte mexicana del golfo de México, Caballero y TelloSandoval (1992) determinaron 14 especies en lenguados de la familia Bothidae y Castillo-Sánchez et al. (1998) también registraron 14 en Paralichthys californicus de Baja California.

Si bien existe alguna información sobre helmintos de peces planos en México, ésta es prácticamente nula para comunidades o a la variación temporal de los parámetros comunitarios. Durante un estudio de distribución de la ictiofauna costera de Campeche fue posible obtener datos sobre la helmintofauna del lenguado Symphurus plagiusa. En consecuencia, los objetivos de este trabajo fueron: describir la variación temporal de la riqueza de especies, número de individuos y similitud cualitativa y cuantitativa de las infracomunidades y comunidades componentes de S. plagiusa, así como determinar posibles asociaciones entre los parámetros fisicoquímicos y los descriptores comunitarios.

\section{Materiales y métodos.}

El estudio se llevó a cabo en 37 estaciones a lo largo de la costa de Campeche y la laguna de Términos (Fig. 1). Esta área es somera, y se caracteriza por una alta productividad biológica, de hidrocarburos y por fuertes descargas estacionales de sus ríos (García-Cuellar et al., 2004). La laguna de Términos está situada en el estado de Campeche, en los 91¹0'00' O y 18²0'19' N (YáñezArancibia y Day,1988), cubre un área de $2500 \mathrm{~km}^{2}$ y comprende las llanuras aluviales del río Usumacinta (Yáñez-Arancibia y Sánchez-Gil, 1986).

Los muestreos se realizaron en la transición entre la temporada de secas y la de lluvias (mayo-septiembre de 2003), obteniendo tamaños de muestra de S. plagiusa entre 42 y 150 ejemplares por mes (Cuadro 1). En el presente trabajo se consideró exclusivamente la variación temporal de los datos y más adelante serán publicados los datos sobre

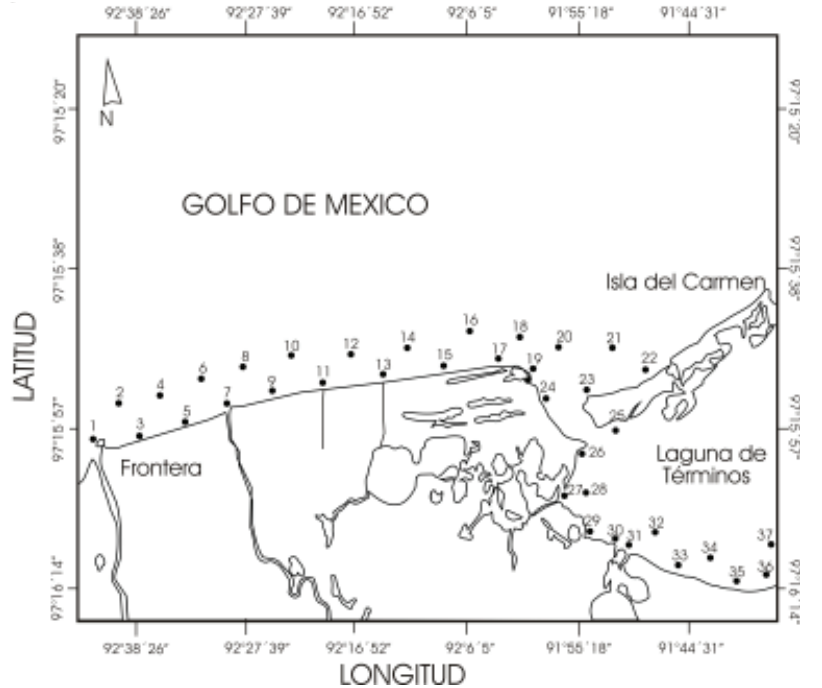

Figura 1. Área de estudio con las 37 estaciones de muestreo en la costa de Campeche y la Laguna de Términos, Campeche, México.

estructura espacial . En cada estación se tomó la posición geográfica por medio de un geoposicionador satelital (GPS), y se registró profundidad $(\mathrm{cm})$, temperatura $\left({ }^{\circ} \mathrm{C}\right)$, salinidad (ups), pH, y concentración de oxígeno (mg/l) por medio de un Hidrolab, y la transparencia $(\mathrm{cm})$ por medio del disco de Secchi.

Los peces se colectaron con una red de arrastre de prueba camaronera de $5 \mathrm{~m}$ de largo, $2.5 \mathrm{~m}$ de abertura y 0.75 pulgadas de luz de malla durante 12 minutos, a una velocidad de 2 nudos cubriendo un área de 2000 $\mathrm{m}^{2}$. Los peces se conservaron en neveras con hielo y se transportaron al laboratorio de Parasitología del CINVESTAV-IPN Unidad Mérida donde se llevó a cabo un examen helmintológico general. De cada pez se tomó longitud total $(\mathrm{cm})$, longitud patrón $(\mathrm{cm})$ y peso (g). Se examinaron piel, escamas, aletas y cavidades en busca de ecto y endoparásitos. Los órganos se separaron individualmente en cajas de Petri con solución salina al $0.07 \%$ y posteriormente se revisaron hígado, riñón, cerebro, bazo, corazón, músculos, mesenterios e intestino por compresión entre 2 vidrios de $10 \mathrm{~cm}$ por lado.

Los individuos de cada especie preliminarmente identificada se preservaron en viales individuales con alcohol al $70 \%$ y fueron etiquetados en la debida forma para su identificación definitiva. Los tremátodos, céstodos y acantocéfalos se tiñeron con paracarmín de Mayer (Vidal-Martínez et al., 2002) y los nemátodos se aclararon en soluciones graduales de glicerina-agua (Moravec et al., 1992). 


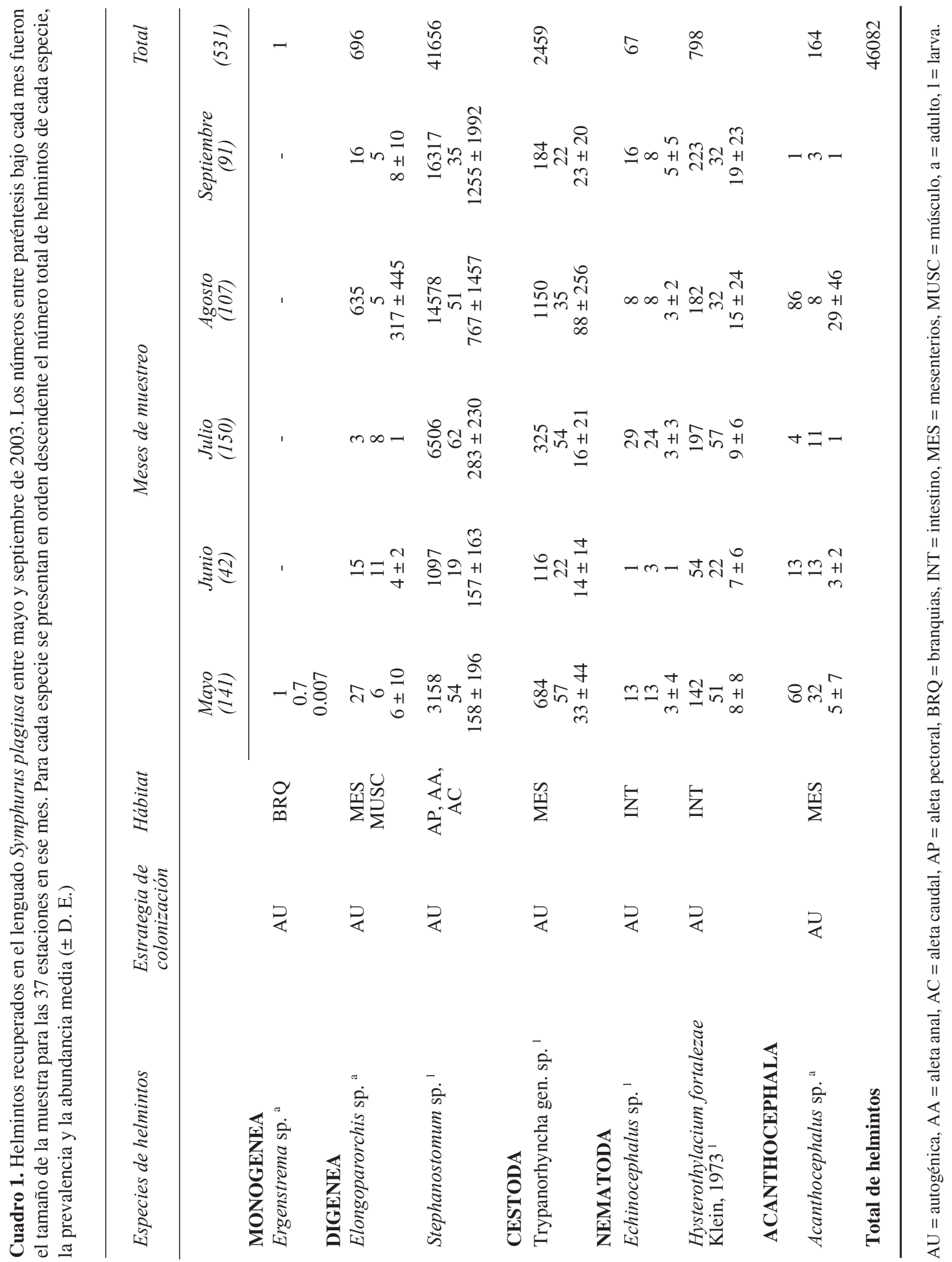


Los parámetros de infección prevalencia y abundancia media se aplicaron siguiendo las recomendaciones de Bush et al. (1997). La prevalencia se calculó dividiendo el número de hospederos infectados con una especie particular de helminto entre el número total de hospederos examinados de una especie (expresada como porcentaje). La abundancia promedio se calculó dividiendo el número total de individuos de una especie particular de helminto entre el total de hospederos examinados de una especie (infectados y no infectados).

En lo que respecta a la estructura de la comunidad, se estudiaron el componente y la infracomunidad (Holmes y Price, 1986). Para el componente, la riqueza fue descrita por el número total de especies de helmintos en la muestra total de hospederos por mes de muestreo; así también se describió el total de helmintos individuales de todas las especies en una muestra de los hospederos por mes de muestreo. Para la infracomunidad, la riqueza de especies se describió mediante el número promedio de especies de helmintos por hospedero examinado por cada mes. Del mismo modo se consideró el número promedio de helmintos por hospedero examinado por cada mes.

Con propósitos comparativos, para describir la diversidad se usó el índice de Simpson, el cual expresa la dominancia o concentración de abundancia de las especies frecuentes dentro de la comunidad en el componente (Krebs, 1989). El promedio del índice de diversidad de Brillouin se utilizó para describir la diversidad de la infracomunidad. Este índice se recomienda para comunidades completamente censadas (donde se conoce el número total de individuos de esa comunidad) y es sensible a la presencia de especies raras (Pielou, 1975). Se utilizó el índice de dominancia no-paramétrico de BergerParker (Magurran, 1988) que se calcula dividiendo el total de individuos de la especie de helminto numéricamente dominante sobre el total de individuos de todas las especies recolectados en un hospedero (nivel infracomunidad) o en la muestra total (nivel componente).

La similitud, para el componente y para la infracomunidad dentro y entre meses, se calculó por medio del índice cualitativo de Jaccard, y cuantitativamente utilizando el porcentaje de similitud (Krebs, 1989). En el componente, la similitud dentro y entre meses fue calculada utilizando los datos del total de especies e individuos colectados en 20 puntos de muestreo seleccionados al azar de cada mes. En la infracomunidad, la similitud dentro y entre meses fue hecha con base en las infracomunidades de helmintos de 20 peces de cada mes seleccionados al azar. El resultado de estas comparaciones se expresó como un valor promedio del índice de similitud de Jaccard entre meses ( \pm D. E.). Se aplicó el mismo procedimiento para el índice de similitud cuantitativo del porcentaje de similitud (Krebs, 1989). Respecto a la predictibilidad en el componente de comunidad (entre el número total de especies e individuos por punto de muestreo de cada mes), así como de las infracomunidades (entre hospederos de diferentes meses), se aceptó que a mayores valores de similitud hubo mayor predictibilidad.

Adicionalmente, las especies se catalogaron como alogénicas o autogénicas (Esch et al., 1990), siendo las primeras las que completan su ciclo de vida en hospederos terrestres (aves y mamíferos), y las especies autogénicas son aquellas que desarrollan por completo su ciclo de vida en ambientes acuáticos.

El supuesto de normalidad en la distribución de los datos se verificó aplicando la prueba de normalidad de Kolmogorov-Smirnov utilizando la aproximación de Lilliefors (Sokal y Rohlf, 1995). En caso de una distribución no normal, los datos fueron transformados a logaritmo natural de $x+1$. La homogeneidad de varianzas fue verificada con la prueba de Bartlett. En aquellos casos en los que no pudieron cumplirse los supuestos del análisis de varianza (ANOVA), se aplicaron pruebas no paramétricas (Kruskal-Wallis). El efecto del factor talla de los hospederos sobre la riqueza y abundancia fue evaluado mediante ANOVA paramétrico de una vía; y controlado por medio de análisis de covarianza de una vía (ANCOVA) (Sokal y Rohlf, 1995).

Se usaron métodos estadísticos multivariados para determinar posibles asociaciones entre la intensidad de infección de cada especie de parásito y las variables ambientales registradas por cada mes de muestreo. Se aplicaron análisis de redundancia (RDA), a las intensidades de los parásitos utilizando el programa Análisis Canónico de Correspondencias CANOCO (ter Braak y Šmilauer, 1998).

Primero se analizó la longitud del gradiente; es decir, qué tan unimodal es la respuesta de una especie a lo largo de un eje de ordenación, que se mide en número de desviaciones estándar por medio de análisis de correspondencias (DCA) (ter Braak y Šmilauer, 1998). Si el valor del gradiente es mayor que 4, se utiliza análisis canónico de correspondencia y se usa análisis de redundancia (RDA) si el valor es menor que 4 (ter Braak y Šmilauer, 1998). En el caso de nuestros datos, el gradiente fue menor que 4; por tanto, se usó RDA y se da por hecho que las curvas de respuesta de las especies de parásitos con respecto a las variables ambientales pueden ser lineales. Se utilizaron aleatorizaciones de Montecarlo para determinar la significancia de los ejes canónicos para ambos helmintos y variables ambientales con base en 10000 iteraciones entre los 5 meses de muestreo (mayoseptiembre de 2003). Las variables ambientales que se consideraron fueron salinidad (ups), temperatura $\left({ }^{\circ} \mathrm{C}\right), \mathrm{pH}$, 
$\mathrm{O}_{2}(\mathrm{mg} / \mathrm{l})$ y número de peces revisados, obtenidos para cada mes de muestreo entre mayo y septiembre de 2003. Para comparar entre meses de muestreo se utilizaron datos de la descarga de agua del río Usumacinta (aledaño al área de estudio). Se obtuvieron datos mensuales de descarga del río entre mayo y septiembre para los años 1947-1983 de la UNESCO (Fekete et al., 2000). Los datos de descarga se obtuvieron en $\mathrm{m}^{3} / \mathrm{s}$ por mes. Los datos del número de helmintos se transformaron a distancia Hellinger según Legendre y Gallagher (2001). El mismo procedimiento se llevó a cabo para las variables biológicas del hospedero (número, longitud total (LT), longitud patrón (LP) y peso). La significancia de los estadísticos usados fue determinada a $\mathrm{p}<0.05$.

\section{Resultados}

De los 531 hospederos examinados en las 37 estaciones para los 5 meses de muestreo, se obtuvo un total de 46 118 helmintos parásitos pertenecientes a 8 especies en $S$. plagiusa.

El Phylum Nematoda fue el mejor representado con 3 especies, seguido de la clase Digenea (2 especies) y finalmente las clases Monogenea, Cestoda y el Phylum Acanthocephala con 1 especie (Cuadro 1).

El Cuadro 1 muestra la composición de especies de helmintos registrada en $S$. plagiusa en los 5 meses de muestreo, así como el número total de individuos, la prevalencia y la abundancia promedio para cada especie. Las especies más prevalentes y abundantes durante los 5 meses fueron Stephanostomum sp. (19\% en junio a $62 \%$ en julio) y Trypanorhyncha gen. sp. (22\% en junio a $47 \%$ en mayo). En cuanto a la abundancia, Stephanostomum sp. fue la especie numéricamente dominante en estas comunidades con valores de $157 \pm 163$ en junio a $1255 \pm 1992$ individuos en septiembre, seguida de Trypanorhyncha gen. sp. (14 \pm 14 en junio a $88 \pm 256$ en agosto) y de $H$. fortalezae $(7 \pm$ 6 en junio a $19 \pm 23$ en septiembre). Elongoparorchis sp. tuvo valores bajos de prevalencia a lo largo del estudio, pero fue muy abundante entre agosto y septiembre 2003 (Cuadro 1).

En el Cuadro 2 se muestran los parámetros de las comunidades de helmintos de $S$. plagiusa en el componente de comunidad. El número total de especies de helmintos varió entre 7 y 8 , siendo mayor únicamente en mayo, debido a la presencia de Ergenstrema sp. El número total de helmintos varió entre 1315 y 16775 en junio y septiembre respectivamente. Los valores de diversidad por el índice de Simpson estuvieron entre 0.98 y 1 durante los 5 meses de estudio. Claramente, la equidad tuvo valores entre 0.27 y 0.37 , y la dominancia numérica expresada por Berger-Parker entre 0.98 y 1 . La metacercaria de Stephanostomum sp. fue la especie numéricamente

Cuadro 2. Descriptores a nivel comunidad componente de helmintos del lenguado Symphurus plagiusa de la costa de Campeche y la Laguna de Términos entre mayo y septiembre de 2003

\begin{tabular}{|c|c|c|c|c|c|}
\hline & Mayo & Junio & Julio & Agosto & Septiembre \\
\hline $\begin{array}{l}\text { Número total de } \\
\text { especies }\end{array}$ & 8 & 7 & 7 & 7 & 7 \\
\hline $\begin{array}{l}\text { Número total de } \\
\text { helmintos }\end{array}$ & 4208 & 1315 & 7119 & 16701 & 16775 \\
\hline $\begin{array}{l}\text { Diversidad } \\
\text { (Simpson) } \\
\text { Equidad } \\
\text { Dominancia } \\
\text { (Berger-Parker) }\end{array}$ & $\begin{array}{l}0.98 \\
0.37\end{array}$ & $\begin{array}{l}0.99 \\
0.30\end{array}$ & $\begin{array}{c}1 \\
0.33\end{array}$ & $\begin{array}{l}0.98 \\
0.27\end{array}$ & $\begin{array}{c}1 \\
0.33\end{array}$ \\
\hline $\begin{array}{l}\text { Especie } \\
\text { dominante }\end{array}$ & $\begin{array}{l}\text { Stephanostomum } \\
\text { sp. }\end{array}$ & $\begin{array}{l}\text { Stephanostomum } \\
\text { sp. }\end{array}$ & $\begin{array}{l}\text { Stephanostomum } \\
\text { sp. }\end{array}$ & $\begin{array}{l}\text { Stephanostomum } \\
\text { sp. }\end{array}$ & $\begin{array}{l}\text { Stephanostomum } \\
\text { sp. }\end{array}$ \\
\hline $\begin{array}{l}\text { Estrategia de } \\
\text { colonización }\end{array}$ & $\mathrm{AU}$ & AU & $\mathrm{AU}$ & $\mathrm{AU}$ & $\mathrm{AU}$ \\
\hline $\begin{array}{l}\text { Status } \\
\text { (Especialista/ } \\
\text { Generalista) }\end{array}$ & $\mathrm{G}$ & $\mathrm{G}$ & $\mathrm{G}$ & $\mathrm{G}$ & $\mathrm{G}$ \\
\hline
\end{tabular}

$\mathrm{AU}=$ autogénica, $\mathrm{G}=$ generalista. 
dominante durante todo el estudio, siendo ésta autogénica y generalista. La figura 2 presenta la similitud tanto cualitativa como cuantitativa dentro y entre meses tanto para el componente de comunidad e infracomunidades. Para el componente, la similitud cualitativa dentro de meses tuvo valores de $0.03 \pm 0.13$ para junio a $0.25 \pm$ 0.34 para julio (Fig. 2a). La similitud cuantitativa dentro de meses tuvo valores de $27 \pm 37$ para mayo a $51 \pm 49$ para junio (Fig. 2b). La similitud cualitativa entre meses tuvo valores mínimos de $0.37 \pm 0.43$, comparando junio y septiembre, a $0.63 \pm 0.28$, comparando julio y agosto (Fig. 2c). La similitud cuantitativa entre meses tuvo valores de $72 \pm 17 \%$, comparando julio con agosto, a $82 \pm 15$ entre junio y julio (Fig. 2d).

En el Cuadro 3 se muestran los descriptores de las infracomunidades. El número promedio de especies por hospedero varió de 2 a 3 . Y el número promedio de helmintos por hospedero estuvo entre $12 \pm 8.70$ y $47 \pm$ 31.81; siendo este último valor, el mayor para el mes de septiembre. La diversidad según el índice de Brillouin presenta el valor más alto en las infracomunidades del mes de julio. El valor de la equidad varió de $0.02 \pm 0.01$ a 0.30 \pm 0.21 . El valor de dominancia mayor según la proporción de las especies fue el de Stephanostomum $\mathrm{sp}$. $(0.88 \pm 0.25)$ en septiembre.

Para la infracomunidad, la similitud cualitativa dentro de meses tuvo valores de $0.43 \pm 0.24$ para mayo y entre $0.61 \pm 0.25$ a $0.59 \pm 0.24$ entre julio y septiembre (Figura 2e). La similitud cuantitativa dentro de meses tuvo valores entre $28 \pm 24$ para mayo y entre $46 \pm 24$ a $45 \pm 29$ entre julio a septiembre (Fig. 2f). La similitud cualitativa entre meses tuvo valores entre $0.66 \pm 0.32$ a $0.75 \pm 0.21$, incluyendo mayo y junio. Sin embargo, en comparaciones entre julio, agosto y septiembre se alcanzaron valores de $0.87 \pm 0.13$ a $0.89 \pm 0.10$ (Fig. 2 g). La similitud cuantitativa entre meses tuvo valores de $59 \pm 28$ a $70 \pm 24$, incluyendo mayo y junio. Al comparar sólo entre julio, agosto y septiembre los valores se mantuvieron entre $74 \pm 25$ a $77 \pm 21$ (Fig. $2 h)$.

Debido a que hubo diferencias significativas en la longitud total de los peces (ANOVA: $\mathrm{F}_{4},{ }_{517}=8.80, \mathrm{P}<$ 0.05), longitud patrón (ANOVA: $\mathrm{F}_{4},{ }_{517}=11.37, \mathrm{P}<0.05$ ) y peso total (ANOVA: $\mathrm{F}_{4},{ }_{517}=7.71, \mathrm{P}<0.05$ ) entre meses, se realizaron ANCOVAS, usando como covariable la longitud total o el peso, según el caso, para controlar el efecto de éstos sobre la riqueza de especies y abundancia. No se observaron diferencias significativas en el número promedio de especies de helmintos por pez entre meses $\left(\mathrm{F}_{4,516}=0.18 ; \mathrm{p}>0.05\right)$, mientras que estas diferencias si se observaron para los valores promedio del número de helmintos entre meses (ANCOVA $F_{4,516}=28.03$; $\mathrm{p}<0.05$ ).

Dentro de cada mes, no hubo asociaciones significativas entre el número de helmintos de alguna especie y los valores de los parámetros fisicoquímicos para ninguno de los ejes canónicos en el análisis de redundancia (RDA) (mayo: F $=2.796, \mathrm{P}=0.454$; junio: $\mathrm{F}=0.790, \mathrm{P}=0.208$; julio: $\mathrm{F}=$ 1.865, $\mathrm{P}=0.796$; agosto: $\mathrm{F}=2.494, \mathrm{P}=0.448$ y septiembre: $\mathrm{F}=0.946, \mathrm{P}=0.941$ ). Sin embargo, hubo diferencias significativas utilizando el número de helmintos de cada especie por punto de muestreo entre meses como variable biológica y la descarga de agua del río Usumacinta en $\mathrm{m}^{3} / \mathrm{s}$ como variable ambiental, tanto para el primer eje canónico ( $\mathrm{F}=8.464 ; \mathrm{P}=0.0114)$, como para los 4 ejes canónicos en conjunto $(\mathrm{F}=2.259 ; \mathrm{P}=0.0088)$ controlando el efecto de las covariables peso y longitud estándar de los peces. La figura 3 muestra las asociaciones entre el número de helmintos de cada especie (flechas sólidas) y la descarga de agua del río Usumacinta en los meses de muestreo (flechas punteadas). Para interpretar este gráfico, es importante considerar que a mayor longitud de una flecha mayor es el porcentaje de varianza con que contribuye esa variable y cuanto menor es el ángulo entre las variables biológicas y ambientales, mayor es su asociación estadística. Además, si las flechas van en la misma dirección, las asociaciones son positivas y si van en contra son negativas. Dado esto, Stephanostomum sp. fue muy abundante a partir de julio y hasta septiembre, y su abundancia aparece asociada positivamente a las descargas del río en esos meses. Trypanorhyncha gen. sp. aparece asociado positivamente a las descargas de mayo (Fig. 3). Sin embargo, la mayoría de las especies estuvieron asociadas negativamente con las descargas en julio, agosto y septiembre.

En el caso de las variables morfométricas de S. plagiusa, al igual hubo asociaciones significativas entre el número de peces por punto de muestreo y los datos de las descargas. Esto sucedió tanto para el primer eje canónico $(\mathrm{F}=12.286$, $\mathrm{P}=0.0244)$ como para los 4 ejes $(\mathrm{F}=2.479, \mathrm{P}=0.0264)$. La figura 4 muestra que hubo asociaciones negativas entre el número de peces (número de hospederos), la longitud total (LT), patrón (LP) y el peso con respecto a la descarga del río entre junio a septiembre.

\section{Discusión}

Las comunidades de helmintos de S. plagiusa, independientemente del mes de muestreo, presentaron valores intermedios de riqueza de especies, fueron poco diversas y con alta dominancia numérica, aunque con un nivel de predictibilidad alto, en comparación con otros peces planos estudiados en México y otras partes del mundo (Cuadros 4 y 5). El número de individuos fue elevado en todos los meses debido principalmente a la dominancia numérica de la metacercaria de Stephanostomum sp. 
a)

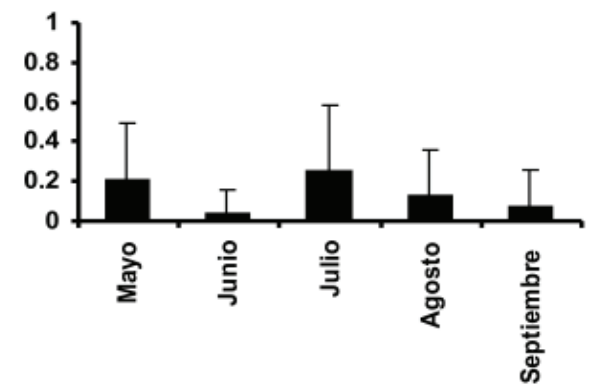

c)

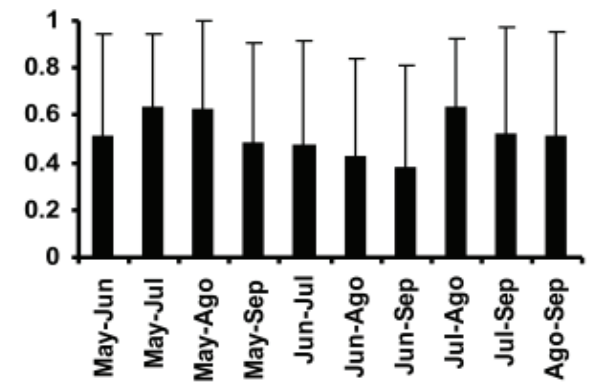

e)

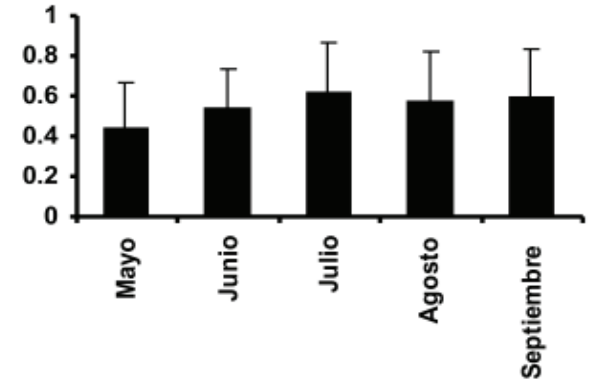

g)

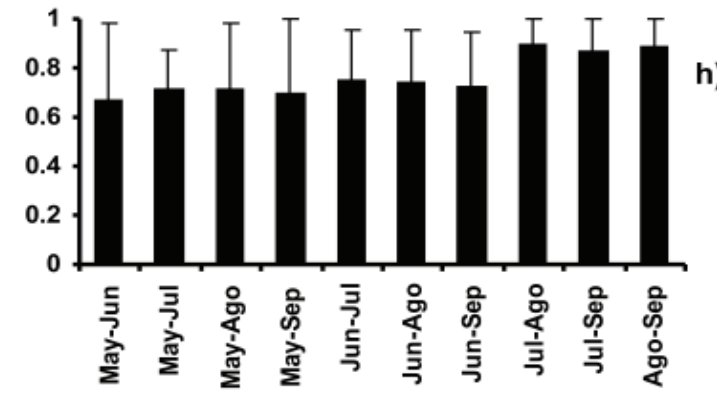

b)

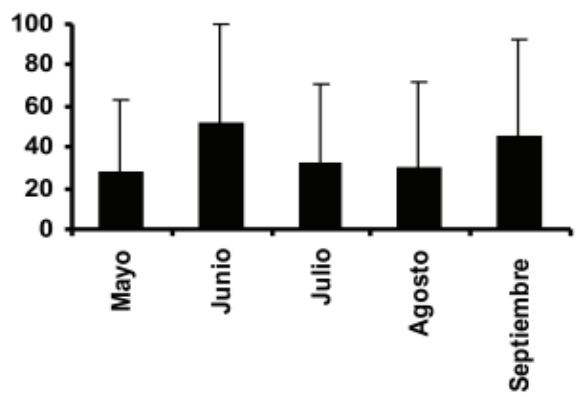

d)

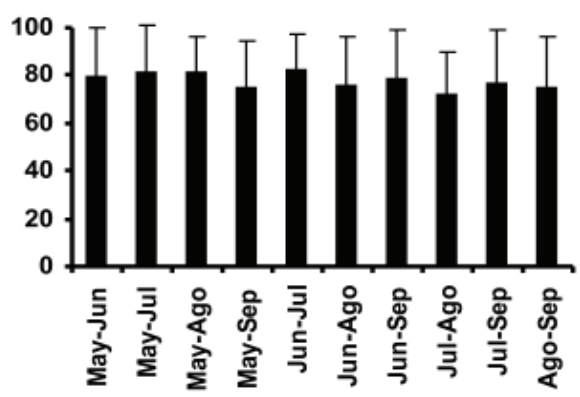

f)

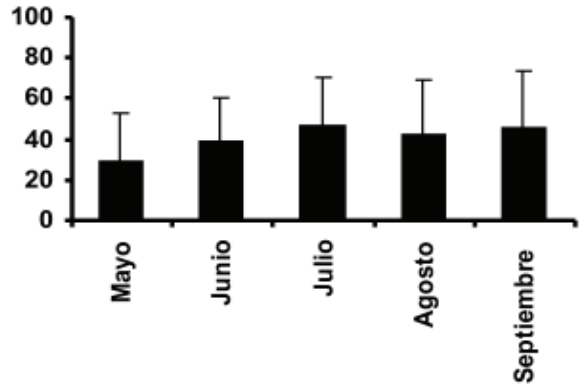

h)

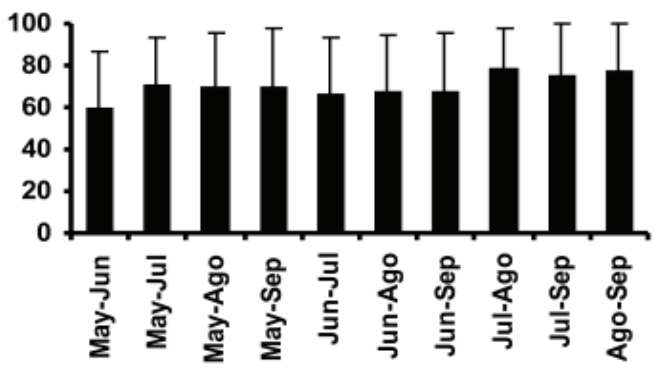

Figura 2. a, índices de similitud cualitativa (coeficiente de Jaccard) para componente de comunidad dentro de cada mes; b, índices de similitud cuantitativa (porcentaje de similitud) para componente de comunidad dentro de cada mes; c, índices de similitud cualitativa para componente de comunidad entre meses; $d$, índices de similitud cuantitativa para componente de comunidad entre meses. La desviación estándar en comunidades componentes resultó de la variación de los valores de similitud de las 37 estaciones de muestreo dentro de cada mes y de la comparación de 20 estaciones de muestreo por cada mes para la comparación entre meses; e, índices de similitud cualitativa para infracomunidad dentro de cada mes; $f$, índices de similitud cuantitativa para infracomunidad dentro de cada mes; g, índices de similitud cualitativa para infracomunidad entre meses; h, índices de similitud cuantitativa para infracomunidad entre meses. La desviación estándar en infracomunidades resultó de la variación de las infracomunidades de todos los peces registrados para las 37 estaciones de muestreo dentro de meses. Para la comparación entre meses, se llevó a cabo la comparación de 20 infracomunidades tomadas al azar de los 37 puntos de muestreo en un mes con otras 20 infracomunidades de otro mes. 
Cuadro 3. Descriptores de las infracomunidades de helmintos del lenguado Symphurus plagiusa de la costa de Campeche y la Laguna de Términos entre mayo y septiembre de 2003. La desviación estándar se abrevió como ( \pm D. E.)

\begin{tabular}{|c|c|c|c|c|c|}
\hline & Mayo & Junio & Julio & Agosto & Septiembre \\
\hline $\begin{array}{l}\text { Promedio de especies } \\
\text { por hospedero } \\
( \pm \text { D. E. })\end{array}$ & $2 \pm 0.73$ & $2 \pm 0.93$ & $2 \pm 0.55$ & $2 \pm 0.55$ & $3 \pm 0.66$ \\
\hline $\begin{array}{l}\text { Promedio de helmintos } \\
\text { por hospedero } \\
( \pm \text { D. E.) }\end{array}$ & $19 \pm 16.66$ & $12 \pm 8.70$ & $24 \pm 20.75$ & $22 \pm 21.05$ & $47 \pm 31.81$ \\
\hline $\begin{array}{l}\text { Diversidad } \\
\text { Brillouin's }( \pm \text { D. E.) } \\
\text { Equidad }( \pm \text { D. E.) }\end{array}$ & $\begin{array}{l}0.01 \pm 0.01 \\
0.02 \pm 0.01\end{array}$ & $\begin{array}{l}0.22 \pm 0.06 \\
0.30 \pm 0.21\end{array}$ & $\begin{array}{l}0.24 \pm 0.11 \\
0.26 \pm 0.16\end{array}$ & $\begin{array}{l}0.13 \pm 0.09 \\
0.18 \pm 0.11\end{array}$ & $\begin{array}{l}0.09 \pm 0.12 \\
0.15 \pm 0.12\end{array}$ \\
\hline $\begin{array}{l}\text { Dominancia numérica } \\
\text { Berger-Parker } \\
( \pm \text { D. E.) }\end{array}$ & $0.54 \pm 0.39$ & $0.49 \pm 0.42$ & $0.79 \pm 0.29$ & $0.55 \pm 0.44$ & $0.88 \pm 0.25$ \\
\hline $\begin{array}{l}\text { Especie } \\
\text { numéricamente } \\
\text { dominante }\end{array}$ & $\begin{array}{l}\text { Stephanostomum } \\
\text { sp. }\end{array}$ & $\begin{array}{l}\text { Elongoparorchis } \\
\text { sp. }\end{array}$ & $\begin{array}{l}\text { Stephanostomum } \\
\text { sp. }\end{array}$ & $\begin{array}{l}\text { Elongoparorchis } \\
\text { sp. }\end{array}$ & $\begin{array}{c}\text { Stephanostomum } \\
\text { sp. }\end{array}$ \\
\hline
\end{tabular}

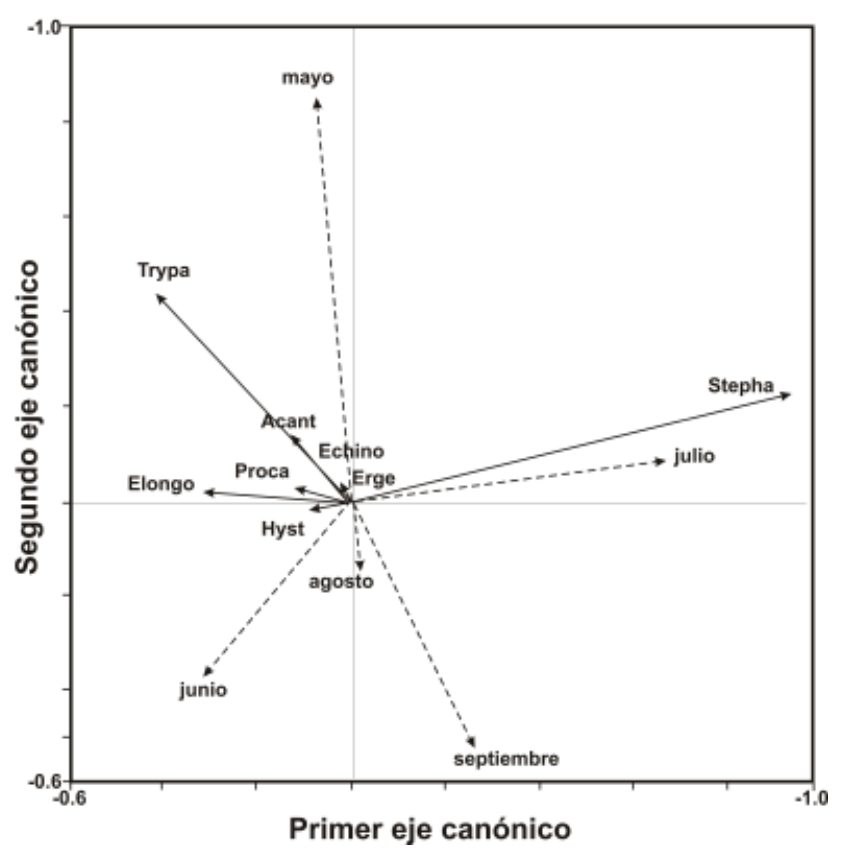

Figura 3. Análisis de redundancia (RDA) donde se muestra la asociación estadística entre el número de helmintos de cada especie y la descarga del río Usimacinta (en $\mathrm{m}^{3} / \mathrm{s}$ ) entre los meses de mayo a septiembre. Los acrónimos empleados fueron los siguientes: Acant $=$ Acanthocephalus sp., Echino $=$ Echinocephalus sp., Elongo = Elongoparorchis sp., Hyst. = Hysterothylacium fortalezae, Erge = Ergenstrema sp., Proca $=$ Procamallanus $($ Spirocamallanus $)$ cricotus, Stepha $=$ Stephanostomum sp., Trypa = Trypanorhyncha gen. sp.
Claramente, el lenguado adquiere gran cantidad de estas formas larvales debido a sus hábitos sedentarios y bentónicos.

De las 8 especies de helmintos recuperados (Cuadro 1), 4 se encontraron en estado larval. En estado adulto se registraron el monogéneo Ergenstrema sp.; el digéneo Elongoparorchis sp., el acantocéfalo Acanthocephalus sp. y el nemátodo Spirocamallanus (Procamallanus) cricotus. La presencia de gran cantidad de estadios larvarios de digéneos sugiere que $S$. plagiusa es un hospedero intermediario o de transporte para estas especies. Los hábitos alimenticios de este lenguado incluyen invertebrados, como jaibas pequeñas, poliquetos, copépodos, anfípodos, ostrácodos, gasterópodos, crustáceos y bivalvos (Vega-Cendejas et al., 1997). Muy probablemente estos organismos son los primeros hospederos intermediarios de las especies observadas. Por tanto, la presencia de la gran cantidad de estados larvarios en este hospedero se explica con base en sus hábitos sedentarios y alimenticios.

Los hospederos definitivos de las especies observadas en $S$. plagiusa son principalmente piscívoros (Bartoli y Bray, 2004). Tal es el caso de delfines, bufeos o toninas que se alimentan de estos peces. El que estos mamíferos marinos se alimenten de peces planos ha sido observado ya anteriormente en las costas del golfo de México (Dyer et al., 1997; Delgado, 2002). Estos factores también han sido propuestos para explicar la presencia en forma larval de estas especies de helmintos en otros lenguados (Bothidae) por Caballero y Tello-Sandoval (1992) en el golfo de México y en Paralichthys californicus por Castillo- 
Cuadro 4. Comparación del número de especies parásitos (por grupo taxonómico) de algunos peces planos (Pleuronectiformes)

\begin{tabular}{|c|c|c|c|c|c|c|c|c|c|c|}
\hline \multirow[t]{2}{*}{ Hospedero } & \multirow[t]{2}{*}{ Localidad } & \multicolumn{8}{|c|}{ Parásitos } & \multirow[t]{2}{*}{ Referencias } \\
\hline & & $\mathrm{H}$ & M & $\mathrm{C}$ & $\mathrm{D}$ & $\mathrm{Ce}$ & $\mathrm{N}$ & A & $\mathrm{n}$ & \\
\hline $\begin{array}{l}\text { Hippoglossoides } \\
\text { platessoides }\end{array}$ & Noreste Atlántico & - & - & - & 7 & 1 & 0 & 2 & 74 & $\begin{array}{l}\text { Arthur y Albert } \\
\text { (1994) }\end{array}$ \\
\hline $\begin{array}{l}\text { Hippoglossus } \\
\text { hippoglossus }\end{array}$ & Noreste Atlántico & - & - & - & 16 & 2 & 2 & 2 & 272 & $\begin{array}{l}\text { Scott y Bray } \\
(1989)\end{array}$ \\
\hline $\begin{array}{l}\text { Reinhardtius } \\
\text { hippoglossoides }\end{array}$ & Noreste Atlántico & - & - & - & 10 & 3 & 1 & 1 & 71 & $\begin{array}{l}\text { Scott y Bray } \\
(1989)\end{array}$ \\
\hline R. hippoglossoides & Noreste Atlántico & 1 & 1 & 2 & 18 & 6 & 7 & 5 & 350 & $\begin{array}{l}\text { Arthur y Albert } \\
\text { (1994) }\end{array}$ \\
\hline Parophrys vetulus & Pacífico norte & 1 & 1 & 3 & 6 & 1 & 4 & 1 & 877 & Olson (1978) \\
\hline Liopsetta putnami & $\begin{array}{c}\text { Estuario Gran } \\
\text { Bahía N. Hamshire }\end{array}$ & 1 & 2 & 2 & 9 & 1 & 2 & 1 & 526 & Burn (1980) \\
\hline $\begin{array}{l}\text { Paralichthys } \\
\text { californicus }\end{array}$ & Pacífico norte & 0 & 0 & 0 & 6 & 1 & 6 & 1 & 102 & $\begin{array}{l}\text { Castillo-Sánchez } \\
\text { et al. (1998) }\end{array}$ \\
\hline P. adspersus & Pacífico sur & 1 & 1 & 4 & 3 & 4 & 6 & 2 & 179 & $\begin{array}{l}\text { Oliva et al. } \\
\text { (1996) }\end{array}$ \\
\hline Platichthys flesus & Mar Báltico & 1 & 0 & 2 & 5 & 3 & 11 & 5 & 200 & Køie (1999) \\
\hline $\begin{array}{l}\text { Hippoglossus } \\
\text { stenolepis }\end{array}$ & Pacífico norte & 1 & 0 & 0 & 9 & 2 & 4 & 3 & 432 & $\begin{array}{l}\text { Blaylock et al. } \\
\text { (1998) }\end{array}$ \\
\hline $\begin{array}{l}\text { Hippoglossina } \\
\text { macrops }\end{array}$ & Pacífico sur & - & 1 & 3 & 1 & 3 & 2 & 1 & 60 & Riffo (1991) \\
\hline H. macrops & Pacífico sur & 1 & 2 & 2 & 1 & 3 & 1 & 2 & 123 & $\begin{array}{l}\text { González et al. } \\
\text { (2001) }\end{array}$ \\
\hline $\begin{array}{l}\text { Symphurus } \\
\text { plagiusa }\end{array}$ & $\begin{array}{l}\text { Campeche, golfo } \\
\text { de México }\end{array}$ & 0 & 1 & 0 & 2 & 1 & 3 & 1 & 531 & Presente estudio \\
\hline
\end{tabular}

$\mathrm{H}=$ hirudineos, $\mathrm{M}=$ monogéneos, $\mathrm{C}=$ crustáceos, $\mathrm{D}=$ digéneos, $\mathrm{Ce}=$ céstodos, $\mathrm{N}=$ nemátodos, $\mathrm{A}=$ acantocéfalos, y n= número de la muestra (Modificado de González et al., 2001).

Sánchez et al. (1998) en Baja California, México. Todas las especies de helmintos fueron autogénicas (Cuadro 1), y es claro que deben serlo para poder transmitirse a un pez con hábitos sedentarios y bentónicos como S. plagiusa.

Con excepción de Stephanostomum sp., la prevalencia del resto de las especies recuperadas de $S$. plagiusa fue variable y sus abundancias medias fueron generalmente bajas para los 5 meses muestreados (Cuadro 1). La alta variabilidad en los parámetros de infección de la mayoría de las especies sugiere a su vez que este hospedero está sujeto al efecto de amplia variabilidad ambiental. Es probable que estos parásitos infecten a S. plagiusa en forma oportunista, ya que todos los géneros involucrados (Cuadro 1) parasitan varias familias de peces y por tanto pueden considerarse generalistas. En este estudio, los parámetros de infección son más bajos que los encontrados en otros peces planos de latitudes templadas (Marcogliese et al., 1998; Valtonen et al., 2001; Marques et al., 2006). Es altamente probable que los parámetros de infección de los helmintos en $S$. plagiusa sean fuertemente estacionales debido a un efecto de "dilución" ocasionado por el incremento en las descargas de agua dulce de los ríos en época de lluvias. La 
Cuadro 5. Cuadro comparativo de las comunidades de algunas especies de peces planos (Pleuronectiformes), donde $(\mathrm{I})=$ infracomunidad,$(\mathrm{CC})=$ comunidad componente, $\mathrm{NE}=$ Número de especies, $\mathrm{NH}=$ Número de helmintos, $\mathrm{D}=\mathrm{Diver}-$ sidad, donde Lile, 1998 corresponde al índice de/ Shannon-Wiener; Siddall et al., 1994 / Simpson; Schmidt et al., 2003 / Shannon-Wiener; Marques et al., 2006 / Brillouin y presente estudio / Simpson (CC), Brillouin (I) ), SC= Similitud cualitativa (Índice de Jaccard), y SC $(\%)=$ Similitud cuantitativa (Porcentaje de similitud)

\begin{tabular}{|c|c|c|c|c|c|c|}
\hline $\begin{array}{c}\text { Hospedero } \\
\text { (Pleuronectiformes) }\end{array}$ & $N E$ & $\mathrm{NH}$ & $D$ & $S C$ & $S C(\%)$ & Referencia \\
\hline $\begin{array}{l}\text { Pleuronectes } \\
\text { flesus (I) }\end{array}$ & 8 & 3146 & $0.65 \pm 0.25$ & - & $30 \pm 18$ & (Lile, 1998) \\
\hline $\begin{array}{l}\text { Hippoglossoides } \\
\text { platessoides (I) }\end{array}$ & 10 & 1141 & $0.46 \pm 0.30$ & - & $24 \pm 25$ & (Lile, 1998) \\
\hline $\begin{array}{l}\text { Glyptocephalus } \\
\text { cynoglossus (I) }\end{array}$ & 6 & 1525 & $0.44 \pm 0.22$ & - & $53 \pm 19$ & (Lile, 1998) \\
\hline $\begin{array}{l}\text { Hippoglossus } \\
\text { hippoglossus (I) }\end{array}$ & 8 & 4679 & $0.35 \pm 0.21$ & - & $41 \pm 23$ & (Lile, 1998) \\
\hline $\begin{array}{l}\text { Hippoglossoides } \\
\text { platessoides (I) }\end{array}$ & 7 & - & $0.50 \pm 0.04$ & $41.67 \pm 7.86$ & $66.0 \pm 6.73$ & (Siddall et al.,1994) \\
\hline $\begin{array}{l}\text { Platichthys flesus } \\
\text { (I) }\end{array}$ & $3-7$ & $5-180$ & $0.12-1.25$ & - & - & (Schmidt et al., 2003) \\
\hline $\begin{array}{l}P . \\
\text { flesus }(C C)\end{array}$ & 30 & 77611 & - & $0.46-0.89$ & $0.45-0.87$ & (Schmidt et al., 2003) \\
\hline $\begin{array}{l}\text { Dicologlossa } \\
\text { cuneata (I) }\end{array}$ & 7 & 439 & 0.37 & - & - & (Marques et al., 2006) \\
\hline $\begin{array}{l}\text { Microchirus } \\
\text { azevia (I) }\end{array}$ & 15 & 214 & 0.42 & - & - & (Marques et al., 2006) \\
\hline $\begin{array}{l}M . \\
\text { variegates (I) }\end{array}$ & 5 & 219 & 0.55 & - & - & (Marques et al., 2006) \\
\hline Solea lascaris (I) & 11 & 1394 & 0.74 & - & - & (Marques et al., 2006) \\
\hline $\begin{array}{l}\text { S. } \\
\text { senegalensis (I) }\end{array}$ & 10 & 317 & 0.58 & - & - & (Marques et al., 2006) \\
\hline S. solea (I) & 6 & 142 & 0.64 & - & - & (Marques et al., 2006) \\
\hline $\begin{array}{l}\text { Synaptura } \\
\text { lusitanica (I) }\end{array}$ & 1 & 1 & 0.47 & - & - & (Marques et al., 2006) \\
\hline $\begin{array}{l}\text { Symphurus } \\
\text { plagiusa (CC) }\end{array}$ & 8 & 16775 & 1 & $0.25 \pm 0.33$ & $51 \pm 49$ & Presente estudio \\
\hline $\begin{array}{l}\text { S. } \\
\text { plagiusa (I) }\end{array}$ & $3 \pm 0.66$ & $47 \pm 31.81$ & $0.24 \pm 0.11$ & $0.61 \pm 0.25$ & $46.28 \pm 24.51$ & Presente estudio \\
\hline
\end{tabular}

variación en las descargas en el río Usumacinta oscila entre la temporada de secas (febrero-mayo) y lluvias (mayoseptiembre) de $605 \mathrm{~m}^{3} / \mathrm{s}$ a $3226 \mathrm{~m}^{3} / \mathrm{s}$ (Fekete et al., 2000). Esto influiría negativamente en el caso de helmintos con un número relativamente bajo de formas infectivas como Echinocephalus sp., o Procamallanus (Spirocamallanus) cricotus (Cuadro 1), pero podría tener un efecto dispersivo en el caso de especies con gran cantidad de formas larvales como Stephanostomum sp.

La comunidad componente de lenguado fue pobre en número de especies comparada con la de otros peces marinos planos, encontrándose en este hospedero sólo 8 especies (Cuadro 4), lo que es independiente de la latitud, ya que en España la comunidad componente de helmintos 
de Platichthys flesus está formada por 13 especies (Álvarez et al., 2002), mientras que en Brasil y Chile, Hippoglossina macrops y Paralichthys adspersus albergan 20 y 22 especies respectivamente (Oliva et al., 1996; González et al., 2001). Al igual, otras especies de peces con hábitos pelágicos presentan en el componente comunidades más ricas en número de especies de helmintos. Tal es el caso del mero rojo Epinephelus morio, en el que se han registrado 18 especies (Vidal-Martínez et al., 1998), el pámpano amarillo Trachinotus carolinus con 14 especies (Sánchez-Ramírez y Vidal-Martínez, 2002) y el pargo mulato Lutjanus griseus con 20 especies (Argáez, 2003).

Al comparar entre los meses del muestreo, claramente no hubo diferencias en los valores de diversidad en la comunidad componente (Cuadro 2). En cambio, sí hubo un incremento conforme los meses transcurrieron, especialmente de junio hacia septiembre, lo que se interpreta como resultado del incremento de la descarga de agua de los ríos, los cuales, a su vez, incrementan la probabilidad de dispersión de las formas larvales (cercarias), debido a su transporte junto con las corrientes. Es precisamente en estos meses (julio a septiembre) en los que la precipitación es mayor y genera una mayor descarga (véase figura 1 en Jiménez-García y Vidal-Martínez, 2005). Soporte para esta explicación lo encontramos en los elegantes resultados de Fingerut et al. (2003a y b). Estos autores demostraron que el aumento en la velocidad de las mareas genera un incremento en la liberación de 4 especies de cercarias de los caracoles Cerithidea californica: Himasthla rhigedana, Parorchis acanthus, Renicola buchanani y Euhaplorchis californiensis. Las cercarias mostraron una tendencia geotrópica positiva para nadar directamente hacia el sedimento en busca de sus hospederos intermediarios. Un patrón similar de incremento en el número de metacercarias de Oligogonotylus manteri en peces mantenidos en jaulas flotantes durante la época de mayor precipitación fue registrado recientemente para Yucatán por JiménezGarcía y Vidal-Martínez (2005). Además, este fenómeno de incremento estacional en el volumen de descarga de los ríos ha sido señalado con anterioridad como la principal razón para cambios en la composición del fitoplancton y en las comunidades de decápodos de la costa de Campeche y del sistema lagunar Pom-Atasta (Gracia, 1991; BarreiroGuemes y Aguirre-León, 1999). En este caso, es clara la probabilidad de que también afectará las formas larvales de Trypanorhyncha gen. sp., coracidios, etc.

La similitud para el componente, tanto cualitativa como cuantitativa, fue inesperadamente baja, lo que puede deberse a que hubo puntos de muestreo en los cuales los peces no tuvieron helmintos; por tanto, al compararlos con otros donde hubo una cantidad relativamente grande de especies e individuos, el resultado fue de bajos valores en los índices. Este punto, que se tratará en un próximo artículo relacionado con la estructura espacial de los presentes datos, genera un problema mayúsculo en interpretaciones que se refieren a la comunidad componente: ¿Cuál es el límite espacial de una comunidad componente?

La solución temporal para la similitud en la comunidad componente dentro de cada mes es considerar únicamente el total de especies colectado por mes (Cuadro 2). Dado que los valores estuvieron entre 7 a 8 especies, la similitud fue muy alta y podría considerarse semejante a la similitud tanto cualitativa como cuantitativa obtenida entre meses para el componente. Cuando solo se considera la variación entre meses respecto al total de especies colectadas en comunidades componentes, el panorama es muy distinto. Se elimina la variación espacial y la similitud tanto cualitativa como cuantitativa se elevan fuertemente a valores entre 0.8 y 1 , y entre 60 y $80 \%$, respectivamente (Figs. 2c y d). La diferencia en infracomunidades en los niveles de similitud tanto cualitativa como cuantitativa entre mayo y junio respecto al resto de los meses (Figs. 2e y h), tiene que ver con el reclutamiento y dispersión de las especies numéricamente dominantes en estas comunidades: Stepanosthomum sp. y Trypanorhyncha gen. sp. Es claro que a partir de julio la similitud fue incrementándose gradualmente tanto dentro como entre las infracomunidades. Esto quiere decir que en cada mes más peces se vieron parasitados por las especies antes mencionadas. Este patrón de alta dominancia numérica y alta similitud es típico de comunidades de helmintos pobres en número de especies (Aguirre-Macedo y Kennedy, 1999). Estos autores describieron las comunidades de helmintos del ostión americano Crassostrea virginica, el cual presenta características semejantes a las registradas en este trabajo para S. plagiusa. En C. virginica del sureste de México (Tabasco), las comunidades de helmintos estuvieron dominadas por la metacercaria de Renicola roscovita y tuvieron muy altos niveles de similitud tanto cuantitativa como cualitativa. Su limitada movilidad es característica común en ambos hospederos; por tanto, es probable que exista una coincidencia entre el periodo de reclutamiento de Stepanosthomum sp. y el incremento en las descargas estacionales de los ríos en la zona, lo que a su vez incrementa la probabilidad de que hospederos poco móviles, como los lenguados, sean parasitados por esta especie.

Las infracomunidades de helmintos de S. plagiusa tuvieron valores de diversidad $(0.01 \pm 0.01$ en mayo a 0.24 \pm 0.11 en julio) (Cuadro 3 ) por debajo de los registrados para otros peces planos en otras regiones geográficas (Cuadro 5), o comparados con otros peces marinos como Labrus merula (diversidad: $1.02 \pm 0.23$ ), Symphodus tinca (0.9 \pm 0.29) (Campos y Carbonell, 1994), Trachinotus 
carolinus $(0.60 \pm 0.39-0.93 \pm 0.38)$ (Sánchez-Ramírez y Vidal-Martínez, 2002) y Epinephelus morio (0.78 \pm 0.41) (Vidal-Martínez et al., 1998). Además, hubo una clara tendencia al decremento de los valores de diversidad, de $0.24 \pm 0.11$ en julio a $0.09 \pm 0.12$ en septiembre (Cuadro 3 ). Esto se explica por el incremento en el número promedio de individuos y en la dominancia numérica de mayo a septiembre. Los resultados sugieren que estos incrementos fueron debidos, a su vez, al incremento en la dispersión de Stephanostomum sp. propiciado por el incremento en las descargas de agua dulce antes mencionadas.

Las infracomunidades de helmintos de S. plagiusa fueron similares en el número promedio de especies $(2 \pm$ $0.55-3 \pm 0.66$ ) (Cuadro 3) con otras especies de peces como Labrus merula (riqueza: $4.17 \pm 0.90$ ), Symphodus tinca (4.15 \pm 1.29) (Campos y Carbonell, 1994) o Leiostomus xanthurus $(6.0 \pm 1.9)$ (Thoney, 1993). Los valores también son similares a los de otros peces tropicales pelágicos, como Trachinotus carolinus ( $4 \pm 1-6 \pm 2$ ) (Sánchez-Ramírez y Vidal-Martínez, 2002), Menticirrhus ophicephalus (4.1 \pm 1.5) (Luque y Oliva, 1999), y Epinephelus morio (4.11 \pm 2.08) (Vidal-Martínez et al., 1998). Adicionalmente, las infracomunidades del lenguado no estuvieron saturadas con especies. El hecho de que los peces capturados se encontraran muy cerca de la costa puede hacer difícil que hospederos definitivos, tales como mamíferos marinos, sean infectados o transporten huevos de helmintos para que se infecten los hospederos intermediarios. Por tal razón, es probable que no se completen muchos ciclos de vida y las comunidades no se saturen en esta zona con influencia dulceacuícola.

El análisis de redundancia (RDA), mostró que las variables ambientales consideradas no tuvieron asociaciones estadísticas significativas con el número de helmintos dentro de cada mes. Sin embargo, las diferencias significativas entre la descarga del río Usumacinta (en $\mathrm{m}^{3} / \mathrm{s}$ ) entre los meses de muestreo y el número de helmintos de cada especie y las variables asociadas a peces claramente sugieren el efecto de "dilución" y dispersión de formas larvales que produce la descarga (Figs. 3 y 4). De hecho, la asociación positiva significativa entre Trypanorhyncha gen. sp. y mayo, y Stephanostomum sp. y julio (Fig. 3), sugieren que en estos meses se da un efecto de dispersión de formas larvales de estas especies hacia $S$. plagiusa. En cambio, el repentino incremento en el volumen de descarga en junio, agosto y septiembre, produce este efecto de dilución de ambos peces y el resto de las especies de helmintos del Cuadro 1. La interpretación final es que tanto peces como helmintos se ven desplazados mar adentro, fuera del área muestreada. Soporte para esta interpretación la encontramos en Durieux et al. (2007), quienes encontraron que el volumen

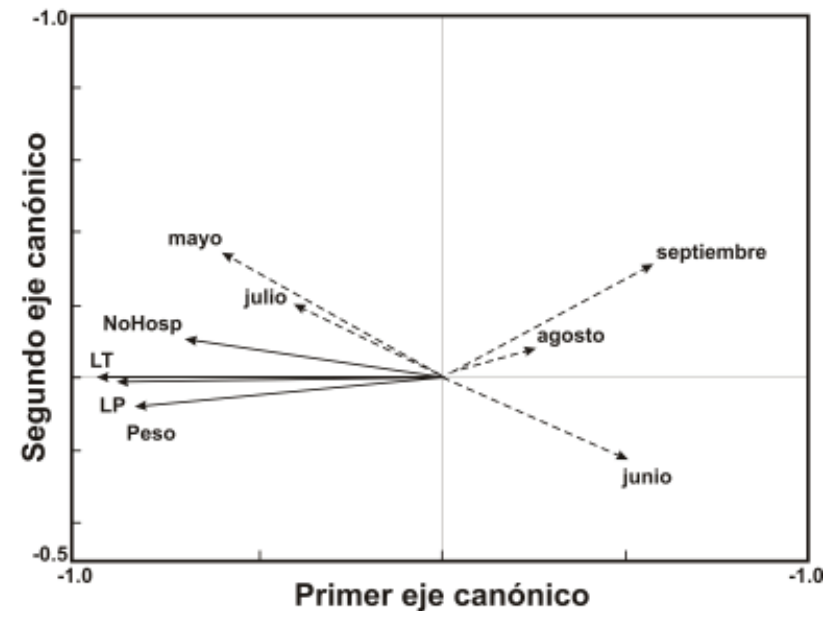

Figura 4. Análisis de redundancia (RDA); donde se muestra la asociación estadística entre las variables morfométricas de los peces $($ No Hosp= número de hospedero, $\mathrm{LT}=$ longitud total, $\mathrm{LP}=$ longitud patrón) y peso con respecto a la descarga de agua del río Usumacinta $\left(\mathrm{en}^{3} / \mathrm{s}\right)$ entre meses.

de descarga de los ríos en la costa atlántica francesa es la principal explicación para el decremento en los parámetros de infección por metacercarias en el pez plano Solea solea. Esto finalmente redunda en un alejamiento de los peces y las formas infectivas que produce una baja en los parámetros de infección.

El hecho de que el mar sea un medio continuo y con mayor energía calórica en los trópicos que en las zonas templadas ha sido sugerido como explicación para una mayor riqueza en número de especies e individuos en latitudes tropicales. Según Rhode (1992), eso conduciría a un mayor consumo de alimento y por tanto, a una mayor probabilidad de infección y transmisión, con el consecuente incremento en los parámetros de infección de las especies tropicales en comparación con las especies encontradas en zonas templadas. Sin embargo, esta hipótesis no aplica del todo en el caso de S. plagiusa. De hecho, es posible concluir que las comunidades de helmintos de S. plagiusa, tanto del componente como de la infracomunidad, presentan valores intermedios en número de especies y alta dominancia numérica. Adicionalmente, factores estacionales como el incremento en las descargas de los ríos influyen positivamente en la transmisión de algunas especies de helmintos como Stephanostomum sp. o Trypanorhyncha gen. sp., pero negativamente sobre todas las otras especies. En consecuencia, consideramos prematuro hacer generalizaciones acerca de la supuesta 
riqueza de las comunidades de helmintos de peces marinos tropicales sin contar con un mayor número de estudios sobre su variación estacional.

\section{Agradecimientos}

Este artículo fue parte de la tesis de licenciatura y forma parte de la tesis de maestría de ARG realizada en el CINVESTAV-IPN, Unidad Mérida. Los autores agradecen el apoyo logístico para la obtención de los lenguados a la Dra. Julia Ramos Miranda de EPOMEX-Universidad Autónoma de Campeche y al proyecto "Caracterización ecológica, biológica, pesquera y social del camarón siete barbas (Xiphopenaeus kroyeri) de la costa sur del Golfo de México. SISIERRA-CONACYT, clave: 20020202, y el apoyo económico de la Gerencia de Seguridad Industrial y Protección Ambiental RMNE, PEMEX Exploración y Producción a través de los contratos no. 4120028470, Impactos antropogénicos sobre el recurso camarón en la Sonda de Campeche, Caracterización de agua de lastre, Cayo Arcas 2005 (contrato No. 412005814) para ARG. También agradecemos a Clara Vivas-Rodríguez, Gregory Arjona-Torres, Trinidad Sosa-Medina, Ana María SánchezManzanilla de CINVESTAV-IPN Unidad Mérida por su ayuda en la revisión e identificación helmintológica, y en el trabajo de campo. Así también a la Dra. Ma. Leopoldina Aguirre-Macedo de CINVESTAV-IPN Unidad Mérida por la identificación de los digéneos, al Dr. David GonzálezSolís de ECOSUR Unidad Chetumal por la identificación de los nemátodos, al Dr. Edgar Mendoza Franco por su valiosa colaboración en la revisión del manuscrito, al Dr. Guillermo Salgado Maldonado de la UNAM por su ayuda en la identificación de acantocéfalos y al Dr. David Cone de la Universidad St. Mary’s Halifax, Canadá por la identificación del monogéneo.

\section{Literatura citada}

Aguirre-Macedo, M. L. y C. R. Kennedy. 1999. Diversity of metazoan parasites of the introduced oyster species Crassostrea gigas in the Exe estuary. Journal of the Marine Biological Association of the United Kingdom 79:57-63.

Álvarez, F., R. Iglesias, A. I. Parama, J. Leiro y M. Sanmartin. 2002. Abdominal macroparasites of commercially important flatfishes (Teleostei: Scophthalmidae, Pleuronectidae, Soleidae) in northwest Spain (ICES IXa). Aquaculture 213:31-53.

Argáez, G. N. 2003. Comunidades de helmintos parásitos intestinales del "pargo mulato" Lutjanus griseus Linneo, 1758 (Pisces: Lutjanidae) en un ambiente marino y uno estuarino del estado de Yucatán, México. Tesis licenciatura Universidad Autónoma de Yucatán, Mérida. 143 p.
Arthur, J. R. y R. Albert. 1994. A survey of the parasites of Greenland halibut (Reinhardtius hippoglossoides) caught of Atlantic Canada, with notes on their zoogeography in this fish. Canadian Journal of Zoology 72:765-778.

Barreiro-Guemes, M. T. y A. Aguirre-León. 1999. Distribución espacio-temporal de la biomasa fitoplánctica en el sistema lagunar Pom-Atasta, Campeche, México. Revista de Biología Tropical 47:27-35.

Bartoli, P. y R. A. Bray. 2004. Four species of Stephanostomum Looss, 1899 (Digenea: Acanthocolpidae) from Seriola dumerli (Risso) (Teleostei: Carangidae) in the western Mediterranean including $S$. euzeti n. sp. Systematic Parasitology 58:41-62.

Blaylock, R. B., L. Margolis y J. C. Holmes.1998. Zoogeography of the parasites of Pacific halibut (Hippoglossus stenolepis) in the northeast Pacific. Canadian Journal of Zoology 76:2262-2273.

Burn, P. R. 1980. The parasites of smooth flounder Liopsetta putnami (Gill) from the Great Bay Estuary, New Hamsphire. Journal of Parasitology 66: 532-541.

Bush, A. O., K. D. Lafferty, J. M. Lotz y A. W. Shostak. 1997. Parasitology meets ecology on its own terms: Margolis et al. Revisited. Journal of Parasitology 83:575-583.

Caballero, G. R. y G. C. Tello-Sandoval. 1992. Tremátodos como indicadores de niveles de contaminación por ácido sulfhídrico en el golfo de México e impacto de este ácido en la salud pública. Anales del Instituto de Ciencias del Mar y Limnología 51:1-6.

Campos, A. y E. Carbonell. 1994. Parasite community diversity in two Mediterranean labrid fishes Symphodus tinca and Labrus merula. Journal of Fish Biology 44:409-413.

Castillo-Sánchez, E., J. Rosales-Casián y G. Pérez-Ponce de León. 1998. Helmintos parásitos de Paralichthys californicus (Osteichthyes: Paralichthydae) en el estero de Punta Banda, bahía de Todos Santos y bahía de San Quintín, Baja California, México. Ciencias Marinas 24:443-462.

Delgado, E. A. 2002. Comparación de parámetros poblacionales de las toninas, Tursiops truncatus, en la región sureste del golfo de México (estados de Tabasco, Campeche, Yucatán y Quintana Roo). Tesis doctorado Facultad de Ciencias, Universidad Nacional Autónoma de México. México, D. F. $160 \mathrm{p}$.

Durieux, E. D. H., R. Galois., M. L. Bégout, P. Sasal y F. Lagardère. 2007. Temporal changes in lipid condition and parasitic infection by digenean metacercarie of young-ofyear common sole Solea solea (L.) in an Atlantic nursery ground (Bay of Biscay, France). Journal of Sea Research 57:162-170.

Dyer, W. G., L. W. Bunkley y E. H. Williams Jr. 1997. Parasites of the dolphinfish (Coryphaena hippurus) in Puerto Rico. Journal of the Helminthological Society of Washington 2:188-194.

Esch, G. W., A. O. Bush y J. M. Aho. 1990. Parasite Communities: Patterns and Processes. Chapman and Hall, London. 335 p.

Fekete, B. M., C. J., Vörösmarty, C. J., Wolfgang. 2000. global, composite runoff fields based on observed river discharge and simulated water balances. Disponible en el URL: hhp://webworld.unesco.org/water/ihp/db/unh_grdc/ 
doc/reportus.pdf

Fingerut, J. T., C. A. Zimmer y R. K. Zimmer. 2003a. Larval swimming overpowers turbulent mixing and facilitates transmission of a marine parasite. Ecology 84:2502-2515.

Fingerut, J. T., C. A. Zimmer y R. K Zimmer. 2003b. Patterns and processes of larval emergence in an estuarine parasite system. Biological Bulletin 205:110-120.

García-Cuellar, A., F. Arreguín-Sánchez, S. Hernández-Vázquez y D. B. Lluch-Cota. 2004. Impacto ecológico de la industria petrolera en la Sonda de Campeche, México, tras tres décadas de actividad: una revisión. Interciencia 29:311-319.

González, M. T., E. Acuña, y M. E. Oliva. 2001. Metazoan parasite fauna of the bigeye flounder, Hippoglossina macrops, from northern Chile. Influence of host age and sex. Memorias do Instituto Oswaldo Cruz 96:1049-1054.

Gracia, A. 1991. Spawning stock-recruitment relationships of white shrimp in the southwestern Gulf of Mexico. Transactions American Fisheries Society 120:519-527.

Holmes, J. C. 1987. The structure of helminth communities. International Journal for Parasitology 17:203-208.

Holmes, J. C. 1990. Helminth communities in marine fishes. In Parasite communities: patterns and processes, G. W. Esch, A. O. Bush y J. M. Aho (eds.). Chapman and Hall, London. p. 101-130.

Holmes, J. C. y P. W. Price. 1986. Communities of parasites. In Community ecology: patterns and processes, D. J. Anderson y J. Kikkawa (eds.). Blackwell, Scientific Publications, Oxford. p. 187-213.

Jiménez-García, M. I. y V. M. Vidal-Martínez. 2005. Temporal variation in the infection dynamics and maturation cycle of Oligogonotylus manteri (Digenea) in the cichlid fish, "Cichlasoma" urophthalmus, from Yucatán, Mexico. Journal of Parasitology 91:1008-1014.

Kennedy, C. R., A. O. Bush, y J. M.. Aho. 1986. Patterns in helminth communities: why are birds and fish so different? Parasitology 93:205-215.

Khan, R. A. 2004. Parasites of fish as biomarkers of environmental degradation: a field study. Bulletin of Environmental Contamination and Toxicology 72:394-400.

Køie, M. 1999. Metazoan parasites of flounder Platichthys flesus (L.) along a transect from the southwestern to the northeastern Baltic Sea. ICES Journal of Marine Science 56:157-163.

Krebs, C. J. 1989. Ecological methodology. Harper Collins, New York, $654 \mathrm{p}$.

Legendre, P. y E .D. Gallagher.2001. Ecologically meaningful transformations for ordination of species data. Oecologia 129:271.280.

Lile, N. K. 1998. Alimentary tract helminths of four pleuronectid flatfish in relation to host phylogeny and ecology. Journal of Fish Biology 53:945-953.

Luque, J. L. y M. E. Oliva. 1999. Metazoan parasite infracommunities of Menticirrhus (Teleostei: Sciaenidae): An amphi-oceanic approximation. Journal of Parasitology 85:379-381.

Magurran, A. E. 1988. Ecological diversity and its measurements. Princeton University Press, Princenton, New Jersey. 179 p.

Marcogliese, D. J. 2001. Pursuing parasites up the food chain: implications of food web structure and function on parasite communities in aquatic systems. Acta Parasitologica 46:82-93.

Marcogliese, D. J., J. J. Nagler y D. G. Cyr. 1998. Effects of exposure to contaminated sediments on the parasite fauna of American plaice (Hippoglossoides platessoides). Bulletin of Environmental Contamination and Toxicology 61:88-95.

Marques, J. F., M. J. Santos y H. N. Cabral. 2006. Soleidae macroparasites along the Portuguese coast: latitudinal variation and host-parasite associations. Marine Biology 150:285-298.

Moravec, F., V. Nasincová y T. Scholz. 1992. Methods of investigation of endoparasitic helminths. Training course of fish parasites. Institute of Parasitology. Academy of Science. Ceske Budejovice, $54 \mathrm{p}$.

Oliva, M. E., R. Castro y R. Burgos.1996. Parasites of the flatfish Paralichthys adspersus (Steindachner, 1867) (Pleuronectiformes) from northern Chile. Memorias do Instituto Oswaldo Cruz 91:301-306.

Olson, R. E.1978. Parasitology of the English sole, Parophrys vetulus Girarg in Oregon USA. Journal of Fish Biology 13:237-248.

Pielou, E. C. 1975. Ecological diversity. Wiley-Interscience, New York. 165 p.

Rhode, K. 1992. Latitudinal gradients in species diversity: The search for the primary cause. Oikos 65:514-527.

Riffo, R. 1991. La fauna de parásitos metazoos del lenguado de ojos grandes Hippoglossina macrops Steindachner, 1876 (Pisces: Bothidae): una aproximación ecológica. Medio ambiente 11:54-60.

Sánchez-Ramírez, C. y V. M. Vidal-Martínez. 2002. Metazoan parasite infracommunities of Florida pompano (Trachinotus carolinus) from the coast of the Yucatan Peninsula, Mexico. Journal Parasitology 6:1087-1094.

Schmidt, V., S. Zander, W. Körting, K. Broeg, V. Westernhagen, H. Dizer, H. Hansen, A. Skouras y D. Steinhagen. 2003. Parasites of flounder (Platichthys flesus) from the German Bight, North Sea, and their potential use in ecosystem monitoring. C. Pollution effects on the parasite community and a comparison to biomarker responses. Helgoland Marine Research 57:262-271.

Scott, J. S. y R. A. Bray. 1989. Helminth parasites of the alimentary tract of Atlantic halibut (Hippoglossus hippoglossus L.) and Greeland halibut (Reinhardtius hippoglossoides Walbaum) on the Scotian Shelf. Canadian Journal of Zoology 67:14761481.

Siddall, R., A. W. Pike y A. H. McVicar 1994. Parasites of flatfish in relation to sewage sludge dumping. Journal of Fish Biology 45:193-209.

Sokal, R. R. y F. J. Rohlf. 1995. Biometry. Third edition. Freeman, New York. 887 p.

ter Braak, C. J. F. y P. Šmilauer. 1998. CANOCO Reference manual and user's guide to Canoco for Windows: software for Canonical Community Ordination (version 4). Microcomputer Power. Ithaca, New York. 352 p.

Thoney, D. A. 1993. Community ecology of the parasites of adult spot. Leiostomus xanthurus, and Atlantic croaker, Micropogonias undulatus (Sciaenidae) in the Cape Hatteras region. Journal of Fish Biology 43:781-804.

Valtonen, E. T., K. Pulkkinen, R. Poulin y M. Julkunen. 2001. 
The structure of parasite component communities in brackish water fishes of the northeastern Baltic Sea. Parasitology 122: 471-481.

Vega-Cendejas M. E., M. Hernández de Santillana y A. G. Cruz. 1997. Los peces de la Reserva de Celestún, Pronatura Península de Yucatán, A.C. The Nature Conservancy. CINVESTAV-IPN, Unidad Mérida, Yucatán. 147 p.

Vidal-Martínez, V. M. y R. Poulin. 2003. Spatial and temporal repeatability in parasite community structure of tropical freshwater hosts. Parasitology 127:387-398.

Vidal-Martínez, V. M., M. L. Aguirre-Macedo, T. Sholz, D. González-Solís y E. F. Mendoza-Franco. 2002. Atlas de los helmintos parásitos de cíclidos de México. Instituto Politécnico Nacional. México, D. F. 183 p.

Vidal-Martínez, V. M., M. L. Aguirre-Macedo, R. C. Vivas-
Rodríguez y F. Moravec. 1998. Las comunidades de macroparásitos del mero Epinephelus morio (Pisces: Serranidae) en la Península de Yucatán, México. Proceedings of the $50^{\text {th }}$ Gulf and Caribbean Fisheries Institute 50:764779 .

Yáñez-Arancibia, A. y J. W. Day Jr. 1988. Ecología de los ecosistemas costeros en el sur del golfo de México: la región de la laguna de Términos. Universidad Nacional Autónoma de México, México D. F. 518 p.

Yáñez-Arancibia, A. y P. Sánchez-Gil.1986. Los peces demersales de la plataforma continental del sur del Golfo de México. 1. Caracterización ambiental, ecología y evaluación de las especies, poblaciones y comunidades. Anales del Instituto de Ciencias del Mar y Limnología. Universidad Nacional Autónoma de México 9:1-230. 